\title{
FRAGMENTOS EPIGRÁFICOS DE GANDUL (ALCALÁ DE GUADAÍRA, SEVILLA)
}

\author{
Antonio Caballos Rufino \\ Universidad de Sevilla \\ caballos@us.es
}

\section{EPIGRAPHICAL FRAGMENTS FROM GANDUL (ALCALÁ DE GUADAÍRA, SEVILLA)}

RESUMEN:Edición de dieciséis fragmentos epigráficos procedentes de Gandul (Alcalá de Guadaíra, Sevilla), verosímil ubicación de la antigua ciudad de Irippo.

PALABRAS CLAVE: Epigrafía, Gandul (Alcalá de Guadaíra, Sevilla), Baetica, Imperio romano.
ABSTRACT: Editing sixteen epigraphic fragments from Gandul (Alcalá de Guadaíra, Sevilla), probable location of the ancient city of Irippo.

KEYWORDS: Epigraphy, Gandul (Alcalá de Guadaíra, Seville), Baetica, Roman Empire.

RECIBIDO: 11.06.2014. ACEPTADO: 25.06.2014

El yacimiento de Gandul $^{1}$, centrado en una meseta que cierra la formación geológica de Los Alcores por el SO, extendido por más de 40 Ha de superficie, en el extremo opuesto a Carmona -distante $21 \mathrm{~km}$ al NE-, próximo al río Guadaíra y a unos $5 \mathrm{~km}$ de la localidad de Alcalá de Guadaíra (provincia de Sevi1la), está ubicado en un punto estratégico de la región, desde el que se domina la campiña, distando unos $22 \mathrm{~km}$ de Hispalis, al NO, y unos 20 de Orippo, al SO. Poblado sin solución de continuidad desde el Calcolítico, con alta significación durante el Bronce final y épocas turdetana y bárquida, como lo demuestra

${ }^{1}$ Somera descripción del yacimiento en el inventario del Patrimonio de Andalucía en la dirección (11.06.14): http://www.iaph.es/patrimonio-inmueble-andalucia/resumen.do?id=i21075. Véase F. Amores, Carta arqueológica de los Alcores, Sevilla 1982, 125. 
la numismática ${ }^{2}$, los restos de la muralla, estructuras emergentes y materiales de superficie permiten caracterizarlo como emplazamiento urbano de importancia en época romana, del que se identifican una necrópolis al norte, donde se localiza un mausoleo romano de comienzos del Imperio ${ }^{3}$, así como restos de un anfiteatro entre éste y el núcleo urbano ${ }^{4}$. La estructura del subsuelo y la ubicación en la cima de la meseta han impedido una significativa acumulación estratigráfica, lo que, junto a la desprotección del yacimiento, viene propiciando un despiadado expolio patrimonial, de lo que quedan múltiples huellas en la superficie.

En tiempos se quiso ubicar en este emplazamiento el municipium Lucurgentum quod Iuli Genius de Plinio ${ }^{5}$, por la identificación en una colección de Alcalá de Guadaíra de una inscripción funeraria, actualmente perdida, de un M. Iunius Brutus lucugert(inus). Hoy, sin embargo, se localiza éste preferentemente en los alrededores de Morón de la Frontera, debido al hallazgo en el Cortijo de Casulillas de la inscripción del sevir lucurgentinus M. Heluius Anthus ${ }^{6}$.

La etimología del nombre del próximo río Guadaíra, sumado a la habitual desinencia prerromana para identificar las ciudades fortificadas y la extraordinaria significación arqueológica del yacimiento han permitido por el contrario a J. Pascual Barea, dada la inexistencia de restos arqueológicos equivalentes en Alcalá de Guadaíra, postular la identificación de Gandul con el antiguo oppidum de Irippo; al que no se refiere Plinio, pero sí está ampliamente documentada por sus acuñaciones ${ }^{7}$. Su categoría municipal, verosímilmente como municipio flavio, se

${ }^{2}$ R. Pliego, "Un campamento cartaginés del siglo IV a.C. en El Gandul (Alcalá de Guadaíra, Sevi1la)", Rivista di Studi Fenici 31 (2003) 31-67; ead., "Un nuevo conjunto monetal cartaginés procedente de El Gandul (Alcalá de Guadaira, Sevilla)", en C. Alfaro, P. Marcos y C. Otero (eds.), XIII Congreso Internacional de Numismática-Madrid 2003-Actas (Madrid 2005) 531-533.

${ }^{3}$ F. Amores y V. Hurtado, "Excavación de un mausoleo circular en Las Canteras (Alcalá de Guadaíra, Sevilla)", Habis 12 (1981) 383-395. Cfr. asimismo V. Hurtado y M. Pellicer, "Excavaciones en La Mesa del Gandul (Alcalá de Guadaíra, Sevilla)", Anuario Arqueológico de Andalucía 1986. Tomo II: Actividades Sistemáticas (Sevilla 1987) 338-341.

${ }^{4}$ J. González (Corpus de Inscripciones Latinas en la provincia de Sevilla, Vol. II, tomo III. La Campiña [Sevilla 1996]; repertorio citado a partir de ahora como CILA, Sevilla) asigna a Alcalá de Guadaíra, identificándola como municipium ignotum, un total de doce epígrafes (CILA, Sevilla $n^{\circ} 915$ a Sevilla $\mathrm{n}^{\circ}$ 926), incluyendo, no sólo los aparecidos en Gandul (CILA, Sevilla n ${ }^{\circ}$ 917, 918, 924 y probablemente $\mathrm{n}^{\circ}$ 921), sino los vinculados o aparecidos en el propio pueblo de Alcalá de Guadaíra y lugares próximos.

${ }^{5}$ NH 3.10. Desde que lo propusiese G. Bonsor (Revue Archéologique 35 [1899] 124 s.) a partir del hallazgo en la próxima Alcalá de Guadaíra de la inscripción CIL II 1264; y así ha sido asumido por otros, como R. Thouvenot (Essai sur la province romaine de Bétique [París 1973²] 194 y 370).

${ }^{6} A E$ 1953, $21=A E$ 1962, $337=C I L A$, Sevilla no 1209. Cfr. J. González Fernández, CILA, Sevilla 263.

${ }^{7}$ J. Pascual Barea, “Irippo y la Mesa de Gandul (Alcalá de Guadaíra): 'la fortificación del río Ira' en época turdetana”, Congreso Internacional Fortificaciones en el entorno del Bajo Guadalquivir (A1calá de Guadaíra 2002) 169-177; id., "La ciudad romana de la Mesa de Gandul como emplazamiento de Iripo y en relación a Lucurgentum y Alcalá de Guadaíra", Actas del III Congreso de Historia Antigua de Andalucía (Córdoba 2001): Andalucía Antigua (Córdoba 2003) 389-407; id., "Comentario a los dáctilos de un fragmento epigráfico de la Mesa de Gandul (Irippo) y su contexto geográfico e histórico”, en J. del Hoyo, C. Fernández, J. Gómez Pallarés y M. Limón (eds.), Ex oficina. Literatura epigráfica en verso (Sevilla 2013) 321- 338. 
fundamenta en la mención epigráfica de honores funerarios públicos ${ }^{8}$; y se confirmaría de leerse en la inscripción CIL II 1263 ordo splen[didissimus Irip]pensium, como propone el mismo J. Pascual Barea'. Por proceder de los alrededores de Alcalá de Guadaíra se ha asignado a esta misma localidad de Irippo la inscripción dedicada por el senador M. Accenna Heluius Agrippa a su hijo homónimo, cuya familia tiene nexos claros con la vecina Hispalis ${ }^{10}$. La misma epigrafía certifica la pervivencia de la ciudad en época visigoda ${ }^{11}$.

Con antelación se habían identificado cuatro inscripciones fragmentarias procedentes de Gandul ${ }^{12}$. Se recoge ahora en el siguiente catálogo ${ }^{13}$ la descripción de los fragmentos encontrados en las prospecciones superficiales llevadas a cabo en el yacimiento por el equipo dirigido por Simon J. Keay en la campaña de $1997^{14}$, así como en algunas visitas posteriores entre dicho año y el de $2002^{15}$, a los que se suma como Addendum otro fragmento aportado por S. Ordóñez Agulla, con la mera pretensión de que, para un yacimiento de tanta significación y desgraciadamente tan inmisericordemente destruido como Gandul, al menos estos humildes restos epigráficos no se pierdan. Se trata de un conjunto de dieciséis piezas, todas ellas simples fragmentos, conteniendo unas pocas letras, si no, en algunos casos, sólo una. Como consecuencia, únicamente en algún caso es posible restituir el sentido original del correspondiente texto o plantear hipótesis sobre su funcionalidad. Como material lapídeo se ha utilizado sin excepción en todos los casos un mármol muy blanco, traslúcido, de grano grueso, en forma de escamas cristalinas, claramente identificables a simple vista; lo que permite asegurar que procede de las canteras de Almadén de la Plata, al norte de la provincia de Sevilla, a aproximadamente unos $80 \mathrm{~km}$ en línea recta al norte de Gandul. La cronología de las piezas abarca desde posiblemente mediados del siglo I hasta el siglo IV. Las primeras catorce fueron entregadas por sus halladores en la

\footnotetext{
${ }^{8}$ CILA, Sevilla no 917 y 918.

9 “Comentario a los dáctilos"..., cit., 332; sobre la inscripción CILA, Sevilla n 916.

${ }^{10}$ CIL II $1262=C I L A$, Sevilla ${ }^{\circ}$ 915. Sobre el personaje, A. Caballos Rufino, Los senadores hispanorromanos y la romanización de Hispania (Siglos IIII). I: Prosopografía, Écija (Sevilla 1990) 27 ss., $\mathrm{n}^{\circ} 1$; y, más recientemente, id., "La presencia hispana en el entorno de Villa Adriana", en R. Hidalgo, P. León (eds.), Roma, Tibur, Baetica. Investigaciones adrianeas (Sevilla 2013) 39. Además de los ya citados, otros epígrafes adscritos a Gandul, de menor significación, son, como ya indiqué, los recogidos en CILA, Sevilla n ${ }^{\circ} 919$ y $921-925$.

${ }^{11}$ CILA, Sevilla ${ }^{\circ} 926$.

${ }^{12}$ CILA, Sevilla no $917,918,921$ y 924.

${ }^{13}$ Estudio llevado a cabo en el marco del Proyecto de I+D+i "La implicación imperial de las elites oriundas de las provincias hispanas y norteafricanas”, del VI Plan Nacional de Investigación Científica, Desarrollo e Innovación Tecnológica (Referencia: HAR2011-29108-C04-01), cofinanciado con fondos FEDER. Agradezco a Armin U. Stylow sus observaciones en relación con este repertorio.

14 "Preliminary investigations at El Gandul, Seville Province, Andalucia", Ciudades Romanas project, interim report No. 1, hasta 2006 disponible en el servidor de Internet de la Universidad de Southampton (http:/www.arch.soton.ac.uk/Research/gandul/Gandul_report.doc).

${ }^{15}$ Agradezco a Simon J. Keay y a José Manuel Rodríguez Hidalgo que me hayan confiado estos materiales para su estudio.
} 
Delegación Provincial de Cultura de la Junta de Andalucía en Sevilla; la última se hallaba en su momento en una colección particular.

1.- Gandul 1997. Roch Pile, E of Site (EG/97/S12). Encontrado en superficie en 1997 por el arqueólogo de la Delegación Provincial de Cultura J. M. Rodríguez Hidalgo.

Fragmento de una muy gruesa placa de mármol blanco rota por todo su perímetro. $(21) \times(8,5) \times 4,5 \mathrm{~cm}$. Reverso liso, pero no perfectamente pulido. La superficie, tanto al anverso como al reverso, se ha visto intensamente sometida a los procesos de erosión, así como alterada por deposiciones calizas. Conserva resto de cinco líneas de texto con una ordinatio muy descuidada, no siendo los renglones ni rectos, ni paralelos entre sí; de tal manera que la desviación relativa entre la segunda línea - la primera que conserva una letra completa - y la tercera llega a ser de unos $45^{\circ}$. Las letras no han sido exactamente grabadas, sino imperfecta y poco profundamente rascadas; dando como resultado el rudimentario procedimiento y el grosor del grano del mármol un surco poco definido, un ductus impreciso y un módulo no homogéneo, causando una impresión de poco cuidado e impericia. Todo ello, unido al fuerte deterioro por rodamiento de la superficie, dificulta sobremanera la identificación de las letras, e impide confirmar plenamente la siguiente transcripción:

$$
\begin{aligned}
& + \\
& +\mathrm{N}+ \\
& + \text { SN } \\
& \text { XSAN } \\
& \text { TT!N }
\end{aligned}
$$

L. 1: De la línea primera línea se conservan sólo dos pequeños trazos oblicuos opuestos, que tal vez pudiesen haber pertenecido a una $\mathrm{A}$, en ese caso muy abierta.

L. 2: De la segunda línea queda, a la izquierda, un pequeño refuerzo horizontal, mientras que de la letra de la derecha resta sólo el hueco redondeado interior, que buenamente pudo corresponder a una $\mathrm{D}$.

L. 3: A comienzos de la tercera, arriba, un trazo inclinado, perteneciente tal vez a una $\mathrm{V}$, debiendo rechazarse una $\mathrm{N}$ por comparación con la de la línea anterior.

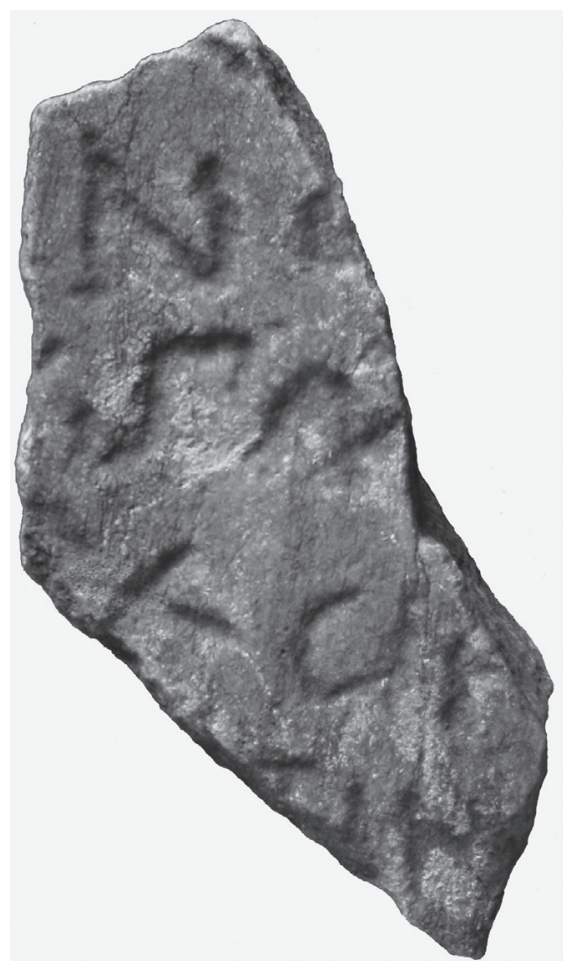

Lámina 1. 
L. 4: En la línea cuarta A. U. Stylow, a quien agradezco su observación, propone restituir [vi]xs(it) an[nnis].

L. 5: T, I y N parece una interpretación adecuada para la última línea. Sería por tanto muy verosímil, en relación con el texto restituido en la línea anterior, desarrollar ...requieui]t in...

Las características materiales y estructurales de la inscripción, así como la forma de las letras, permiten asignarle al epígrafe una cronología de hacia el siglo IV.

2.- Gandul 1997, K9 - 50,0 (EG/97/22).

Fragmento de placa de mármol blanco rota por todo su perímetro. Aristas suavizadas por el rodaje. Reverso desbastado. $(7,7) \times(9,7) \times 2,1 \mathrm{~cm}$.

$+$

$+$

Conserva resto de dos letras en dos líneas superpuestas.

L. 1: Arriba parte inferior, curvada, de una letra que pudo ser D, C, O.

L. 2: Abajo la parte superior de otra letra de gran módulo: posiblemente una $\mathrm{D}, \mathrm{o}$, alternativamente $\mathrm{B}, \mathrm{P}, \mathrm{R}$.

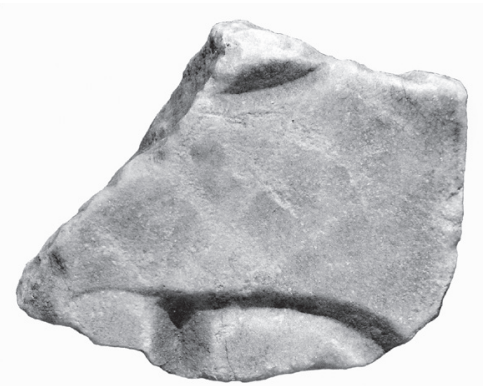

Lámina 2.

3.- Gandul 1997. S6 - Next to section of "Public Buildings wall" (EG/97/S6).

Fragmento de placa de mármol blanco, rota por todo su perímetro, no conservando ningún borde original. Reverso pulido fino. (6) $\mathrm{x}(10) \times 1,6 \mathrm{~cm}$.

$\mathrm{O}$

Conserva sólo parte de una $\mathrm{O}$, grabada con surco en "V" de desigual grosor. La altura original de la letra debió de ser de $6,5 / 7 \mathrm{~cm}$.

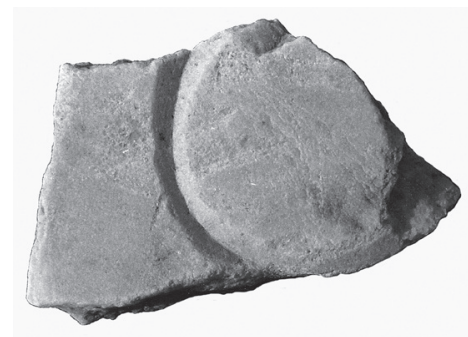

Lámina 3

\section{4.- Gandul 1997. K9 - 75,75 (EG/97/S6).}

Fragmento de placa de mármol blanco, rota por todo su perímetro, no conservando ningún borde original. Reverso pulido fino. $(8,5)$ x (11) x $2,3 \mathrm{~cm}$. Conserva restos de línea de guía de ordinatio incisa abajo, entre las letras. Contiene 
sólo dos letras, grabadas con surco en "V" de desigual grosor.

\section{E+}

A la primera letra, una $\mathrm{E}$, le falta el brazo superior, aunque se ha conservado el módulo: $4 \mathrm{~cm}$ de altura. Brazos anchos, perfectamente horizontales, el central algo más corto, con mínimos refuerzos, que también observamos en el pie del asta vertical. A la derecha de la $\mathrm{E}$,

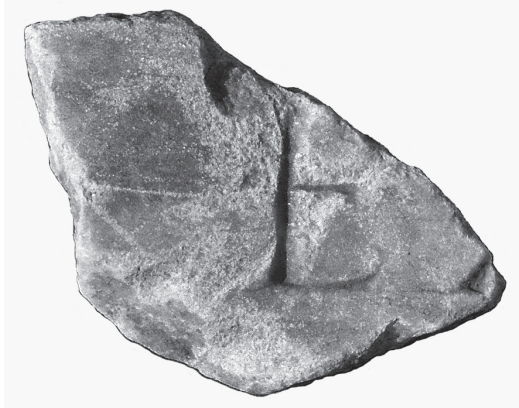

Lámina 4. a $1,7 \mathrm{~cm}$. de ésta, se inicia el refuerzo de un asta que arranca diagonalmente. Debe corresponder bien a una $\mathrm{A}$, a una $\mathrm{M}$ o a una $\mathrm{N}$. Desde la $\mathrm{E}$ hasta el borde izquierdo del fragmento hay $6,7 \mathrm{~cm}$., lo que contrasta con la escasa distancia entre las dos letras. Por ello podemos concluir que la E debe ser la primera letra conservada de la línea. El surco del asta vertical y la desigualdad de los brazos de la E, así como los refuerzos, vienen bien a una cronología del siglo II, sin mayor concreción.

\section{5.- Gandul 1997. K 11 - 0,0 (EG/97/S11).}

Fragmento de placa de mármol blanco, rota por todo su perímetro, no conservando ningún borde original. Reverso pulido fino. $(9,8) \times(6,3) \times 1,8 \mathrm{~cm}$.

\section{M}

Conserva sólo una $\mathrm{M}$ de gran módulo (aprox. 7/7,5 $\mathrm{cm}$ ), de trazos simétricos, identificándose los extremos sólo parcialmente en el bisel. Surco en "V" de grosor variable. Las características formales de la letra permiten asignarle a la inscripción una hipotética cronología de

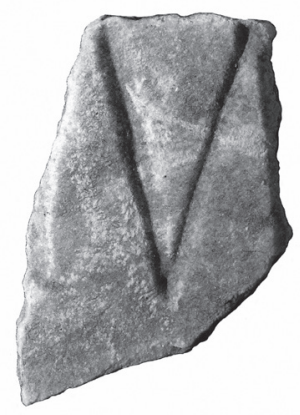

Lámina 5. hacia el siglo II, sin mayor concreción.

6.- Gandul 1997. Fragmento de una inscripción diferente a la anterior, compartiendo la misma referencia K 11 - 0,0 (EG/97/S11), asignada por el equipo arqueológico que la descubrió.

Fragmento de placa de mármol blanco, rota por todo su perímetro, no conservando ningún borde original. Anverso y reverso lisos. La superficie se ha visto muy alterada por la erosión. $(4,5)$ x $(3,8)$ x $1,5 \mathrm{~cm}$. Conserva parte de dos letras, de aprox. 3,5 de módulo. Surco muy fino y poco preciso, que parece más rascado 
que grabado. Ápice en forma de curva y contracurva sobre el asta conservada de la $\mathrm{N}$.

\section{$\mathrm{N}+$}

La segunda letra pudo buenamente ser una B, P, R. Por la tipología de las letras parece hipotéticamente ajustada una cronología del siglo III.

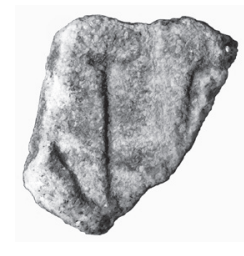

Lámina 6.

7.- Gandul 1997. Sin contexto (EG/97/S17).

Fragmento de placa de mármol blanco, rota por todo su perímetro. $(8,3) \mathrm{x}$ $(7,5)$ x $1,85 \mathrm{~cm}$. Conserva resto de dos líneas.

$\mathrm{E}$

$\cdot$ V

L. 1: En la primera línea se ha conservado la mitad de la letra E: parte del brazo inferior, reforzado, algo del bisel del asta vertical y el ápice del brazo central. $\mathrm{Su}$ surco tiene sección en "V", de desigual anchura. Si la distancia del brazo central a la parte superior fuese la misma que la que hay hasta el pie, entonces el mó-

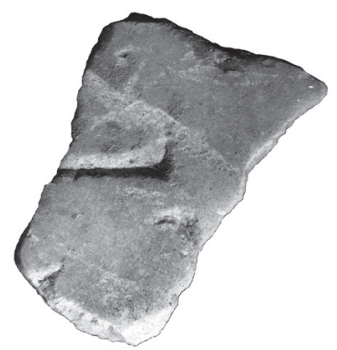

Lámina 7. dulo de las letras en esa línea sería de aproximadamente unos $7,5 \mathrm{~cm}$.

L. 2: La segunda línea, si no se trata de una lasca saltada, comenzaría con una interpunción de sección triangular. A su derecha el refuerzo final y parte de un trazo diagonal, que buenamente pudo haber correspondido a una $\mathrm{V}$.

8.- Gandul 1997. K10 - 50,75 (EG/97/S7).

Fragmento de placa de mármol blanco, rota arriba, izquierda y abajo. Aristas suavizadas por el rodaje. Conserva parte del borde derecho, recto, pulido. Reverso desbastado fino. $(9,1) \times(7) \times 2,1 \mathrm{~cm}$. Del texto únicamente se conserva la parte inferior derecha de una $\mathrm{C}$, de gran módulo, que, a modo de hipótesis, podemos suponer de en torno a los $9 \mathrm{~cm}$. Surco en "V", de grosor variable.

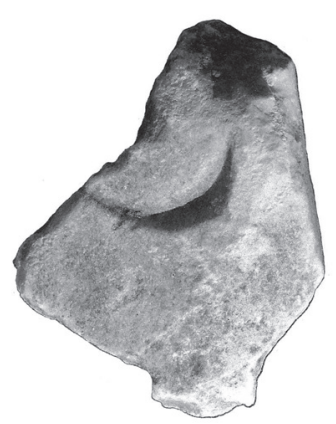

Lámina 8. 
9.- Gandul 1997. K12 - 50,0 (EG/97/S18).

Fragmento de placa de mármol blanco, rota por todo su perímetro. $(4,7) \mathrm{x}$ (8) x 2,3 cm. Conserva resto de dos líneas de texto, separadas entre sí sólo 5/7 $\mathrm{mm}$. Aunque las letras están incompletas, el módulo de las de la primera línea no debió de ser más que de unos aproximadamente $2,5 \mathrm{~cm}^{16}$. El módulo de la segunda línea, mayor, pudo ser de en torno a unos 3,5 cm. Letras de surco en "V", en unos puntos muy ancho, y en otros sólo una fina línea incisa. Los pies de la $\mathrm{M}$ se recurvan hacia fuera.

\section{EM ASC̣V}

L. 1: De la posible E de la primera línea se conserva sólo el brazo inferior. Parece demasiado ancho como para haber correspondido a una $\mathrm{L}$.

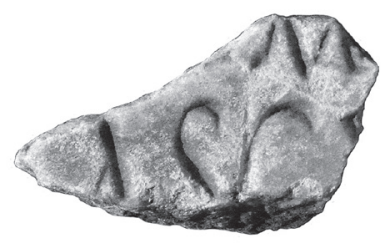

Lámina 9.

L. 2: De la última letra de la segunda línea se identifica sólo el refuerzo de un trazo oblicuo, que, por su inclinación y remate, parece buenamente corresponder a una $\mathrm{V}$, mejor que a una $\mathrm{A}$; quedando las $\mathrm{M}$ y $\mathrm{N}$ por contexto a priori excluidas.

A. U. Stylow sugiere identificar en la segunda línea el cognomen Masculus. Por las características formales de las letras, el epígrafe pudo buenamente datarse en el siglo III.

10.-Gandul 1997. Inscription (Janet's) S8. NSR has note for location.

Fragmento de placa de mármol blanco, rota por todo su perímetro. Reverso pulido. Procesos de alteración en su superficie, tanto al anverso como al reverso. $(7,5) \times(4,5) \times 1,3 \mathrm{~cm}$. Conserva resto de dos letras, incompletas, con surco en "V", de grosor variable. Se identifican $6,3 \mathrm{~cm}$. de un trazo rectilíneo. Por arriba parece apreciarse algo de su refuerzo superior, en forma de fina línea curva, y, en el bisel, aparentemente el vértice de tangencia con el trazo siguiente de la letra. La parte inferior del surco del asta se engruesa, por lo que nos acercamos al refuerzo del pie. Por lo anterior el módulo de las letras debió de ser de aproximadamente 7/7,5 cm.

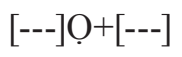

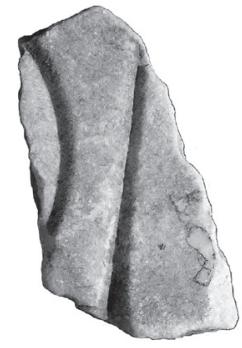

Lámina 10.

${ }^{16}$ La altura dependerá de cuánto sobresalieran los trazos pares de la M sobre el vértice de unión con los impares. 
De la primera letra se conserva una curva, más posiblemente una $\mathrm{O}$ o, como alternativa, una $\mathrm{D}$; quedando excluida una $\mathrm{Q}$ por la proximidad de la letra contigua. El trazo curvo está muy próximo — $9 \mathrm{~mm}$ de distancia mínima - al longitudinal; por esto y por terminar arriba este último, como dije, muy verosímilmente en vértice, debe descartarse que se trate de un asta vertical. Por ello la segunda letra debe ser verosímilmente una $\mathrm{M}$, por la presumible inclinación del trazo, o, como alternativa y de no haber sido así, una N. Parece deba descartarse una A sin travesaño. Con ello quedaría reforzada la identificación de la letra anterior como una $\mathrm{O}$.

\section{1.- Gandul 1997 (EG/97/S10).}

Fragmento de gruesa placa de excepcional factura, de mármol blanco, rota por todo su perímetro. Anverso finísimamente pulido. Reverso pulido, con concreciones calizas. $(16,6)$ x $(15)$ x $3,8 \mathrm{~cm}$. Conserva letras en dos líneas, separadas entre sí 4,1 cm. Sin huellas de la ordinatio. Módulo de las letras: $7 \mathrm{~cm}$. Surco en "V" de desigual grosor. La O más estrecha que una circunferencia. Refuerzos mínimos y pies cortos.

$\stackrel{\mathrm{PO}+}{\mathrm{T} \cdot+}$

L. 1: De la primera letra de la primera línea sólo se ha conservado un posible resto de bisel curvo en la parte superior, por lo que proponemos la posibilidad de identificarla como una P. De la última letra lo único conservado es un asta vertical. Excluida la R por no existir rastro de trazo inclinado, nos quedarían como posibilidades, tanto una I, como tal vez una $\mathrm{N}$, que en este caso sería muy ancha. De pensarse en una $\mathrm{T}$ su trazo horizontal superior tendría que haber sido más estrecho que el de la misma letra conservada en la línea segunda. Por último, de pensarse en una $\mathrm{P}$, entonces habría que suponer que el trazo curvo no se habría unido con el trazo vertical, o lo habría hecho con lo que sería en su momento un finísimo y casi solamente apuntado surco. 
L. 2: En la segunda línea tras la T existe una interpunción triangular con el vértice hacia abajo. La segunda letra conservada de la segunda línea pudo ser una $\mathrm{P} / \mathrm{B} / \mathrm{R}^{17}$.

Puede muy verosímilmente tratarse de una inscripción honorífica por las características formales de la pieza y por el lugar en que fue encontrada, en el corazón de la ciudad, a lo que se suma lo poco del texto conservado, donde resulta posible como hipótesis pensar en restituir en la primera línea, bien pon [tifex?], si no incluso [tribunic(ia)] pot(estate). Por la tipología de las letras podemos asignarle una presumible cronología de mediados o la segunda mitad del siglo I.

\section{2.-Gandul. Sin referencias.}

Fragmento de inscripción de mármol blanco, rota por todo su perímetro. Aristas suavizadas por el rodaje. Picotazo reciente en una esquina y rasguño en el anverso. Reverso pulido fino. $(4,7)$ x $(9,6)$ x 3,8 cm. Posible línea de guía incisa sobre el resto de la letra conservada del segundo renglón, a partir de su derecha, pues a la izquierda el roce reciente ya descrito ha borrado cualquier huella de ordinatio que hubiera podido existir. La separación entre la primera y la segunda línea es de $2,5 \mathrm{~cm}$. Letras de surco en "V", de grosor muy variable.

$+$

$+$

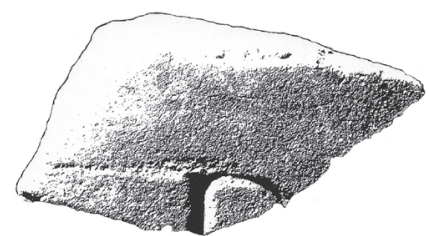

Lámina 12.

L. 1: De la letra de la primera línea se ha conservado un ancho trazo curvo en la base de la letra, bien una $\mathrm{O}$ - aun cuando la curva no forma un arco de circunferencia-, o una $\mathrm{D}$, menos probablemente, por la anchura de la curva, una $\mathrm{B}$. Excluyo una Q por no conservarse, en el lugar correspondiente, el rabo secante de la letra.

L. 2: De la letra de la segunda línea se ha conservado el final, con refuerzo mínimo, de un asta vertical y un arco tangente con ésta. Letra capital sin rasgos de cursividad. Son posibles B, P, R.

Lo poco conservado no nos permite afinar una datación, sin embargo lo descrito nos permite al menos adelantar una cronología del siglo II o hasta el III, sin que nos parezca posible una mayor concreción.

${ }^{17}$ De ser así, la falta de numeral excluiría la posibilidad de [tribunic(ia) po]t(estate). 


\section{3.-Gandul. Sin referencias.}

Fragmento de placa de mármol blanco, rota por todo su perímetro siguiendo el surco de la letra, desigualmente ancho y en forma de "V". $(8,5) \times(6,6) \times 1,3 \mathrm{~cm}$. De la letra se conserva sólo aproximadamente la mitad, por lo que resulta imposible conocer su módulo, aun cuando éste debió de rondar en torno a $\operatorname{los} 15 \mathrm{~cm}$.

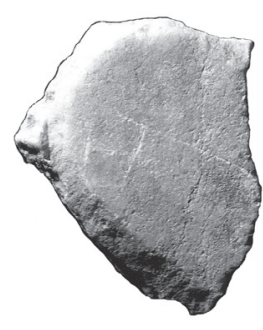

Lámina 13.

\section{S}

Se trata de una $\mathrm{S}$ y no una $\mathrm{O}$, ya que la curva no es homogénea y parece haberse conservado parte del refuerzo final de la letra por su parte superior.

\section{4.- Gandul 2002. Sin referencias.}

Fragmento de placa de mármol blanco, rota por todo su perímetro. Anverso y reverso lisos. Superficie alterada por la erosión, que también ha suavizado los bordes, redondeándolos. $(7,7)$ x $(7,3)$ x 1,9 cm. Conserva sólo parte de una $\mathrm{E}$ o F, de gran módulo, con el asta vertical de surco muy ancho en " $V$ ", que se va engrosando hacia abajo. El brazo horizontal central, más fino, no es perfectamente tangente con el vertical. Con refuerzo ancho.

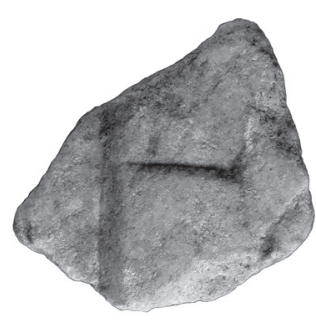

Lámina 14.

\section{5.- Gandul 1997. Tégula con marca. K 10 - 75,50 (EG/97/S14)}

Fragmento de tégula de color rojizo, a horno fuertemente oxidante, con desgrasante fino. Rota a izquierda, arriba y a derecha. Borde plano y perfiles redondeados, tanto hacia el anverso como al reverso. Anverso y reverso lisos. $(8,2) \mathrm{x}$ $(5 / 7,6)$ x 2,8 cm. A 2,1 cm del borde inferior, y paralelo a éste, marca impresa sobre el barro húmedo, sin cartela. Conserva tres letras alargadas, de 1,3 cm de módulo, desigualmente profundas por una impresión no homogénea, y por desgaste de la superficie. Por este mismo motivo, tanto a la derecha como por pérdida de masa a la izquierda, no sabemos si nos ha llegado completo el texto de la marca. La tercera de las letras, grabada tenuemente y parcialmente desaparecida, es más posiblemente una $\mathrm{M}$ que una segunda $\mathrm{N}$, aunque la identificación con esta última letra no puede excluirse tajantemente.

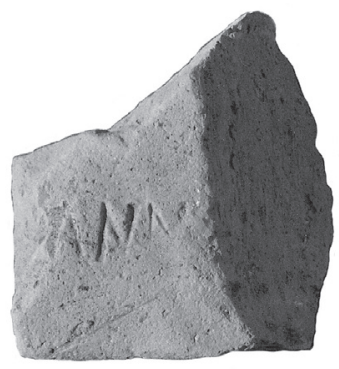

Lámina 15. 


\title{
ADDENDVM: UNA INSCRIPCIÓN MÁS DE GANDUL
}

\author{
Salvador Ordóñez Agulla \\ Universidad de Sevilla \\ sagulla@us.es
}

\section{6.- Gandul 1980.}

Esta pieza, en una colección particular en Sevilla, fue hallada en 1980, en el yacimiento de la Mesa de Gandul. A casi 35 años de distancia, no es posible ya localizar con precisión el lugar concreto de hallazgo, aunque sí se puede acotar lo suficiente como para tener cierta fiabilidad en su procedencia de un área de necrópolis. Más en concreto, la pieza se encontraba entre los materiales exhumados de una excavación clandestina en un punto al norte del sistema defensivo de la Mesa, no lejos del bastión del Toruño, en un ámbito donde los trabajos de prospección del equipo de S. J. Keay ${ }^{18}$ sugieren la existencia, en época romana, de un entorno de funcionalidad artesanal y de enterramientos. En este sentido, cabría relacionar esta pieza con el hallazgo de "occasional fragments of small Latin inscriptions" que, refiere el citado investigador, fueron localizados, junto con restos de escorias, al norte de las defensas más antiguas.

Fragmento de una placa de mármol blanco de grano grueso y brillante, que por sus características externas puede provenir de las canteras de Almadén de la Plata, rota por todo su perímetro, adoptando una forma triangular, sin que se haya conservado ninguno de los bordes originales. A juzgar por lo vivo de las aristas, la pieza apenas ha sufrido rodaje. En el borde superior conserva embutido parte de uno de los vástagos de hierro que fijarían la inscripción a un soporte. $(14,5)$ x $(8,5)$ $\mathrm{x} 1,8-2,4 \mathrm{~cm}$. Anverso y reverso finamente pulidos. Se aprecian manchas de óxido en algunas partes de la superficie, quizá resultado del contacto con las escorias aludidas anteriormente. Conserva restos de cuatro líneas de texto con cuidada ordinatio, no apreciándose líneas de guía. Letras capitales librarias de 3,7 cm de altura, con ductus muy cuidado y grabado con surco en "V" de grosor variable; refuerzos acusados. P con óculo abierto; E con trazos horizontales que apuntan hacia arriba, el primero de los cuales sobresale de la línea de caja; V con trazo derecho en posición casi horizontal; A con travesaño inclinado. Interlineado: 1,5 cm entre 11. 1-2, $2 \mathrm{~cm}$ entre 11. 2-3, $2 \mathrm{~cm}$ entre 11. 3-4. Se ha conservado una interpunción, en 1. 3, triangular con el vértice hacia abajo y la derecha.

${ }^{18}$ S. Keay y D. Wheatley, "Fortificaciones en el Bajo Guadalquivir a finales de la Edad de Hierro y comienzos de la época romana", Congreso Internacional "Fortificaciones en el entorno del Bajo Guadalquivir" (Alcalá de Guadaíra 2002) 98-99. 


\section{$+$ \\ EPVLT \\ $+\mathrm{A} \cdot+$ \\ $+$}

L. 1: Se observa la parte inferior de tres trazos: los de los extremos son pies con sus refuerzos horizontales, en el centro un vértice; puede tratarse de una $\mathrm{M}$.

L. 2: De T se observa en autopsia el extremo izquierdo de su travesaño, del que puede apreciarse claramente que era ondulado.

L. 3: La primera + es parte superior de un trazo vertical $(\mathrm{H}, \mathrm{I}, \mathrm{N}, \mathrm{V})$; La segunda + es parte del bisel izquierdo de un trazo vertical -probablemente una A-, que conserva asimismo los refuerzos superior e inferior.

L. 4: + es refuerzo superior de un carácter.

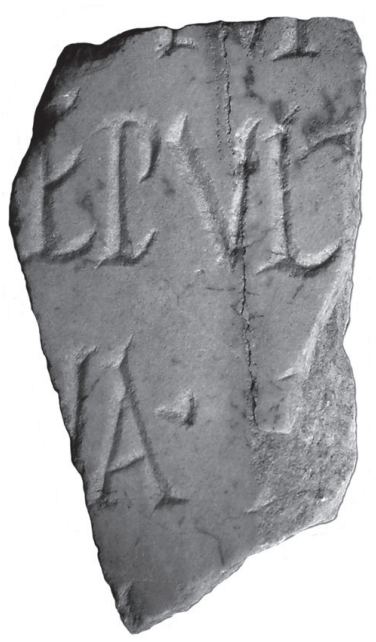

Lámina 16.

Es la línea 2 la única que proporciona algún tipo de información en esta pieza, en el término sepult, que únicamente puede referirse a sepultus-a, sepulti o sepultura. Muy probablemente se trate de una inscripción funeraria, opción preferible, por entorno de proveniencia, tipología y cronología, a considerar un eventual homenaje municipal con mención del locus sepulturae y los otros honores usuales. A tenor del tipo de letra empleado, la cronología de este fragmento puede situarse a fines del siglo II d.C. 